\title{
Blocking in children from two socioeconomic levels
}

\author{
JEAN L. BRESNAHAN \\ Herbert H. Lehman College, City University of New York, Bronx, New York 10468
}

and

\author{
MARGARET ANN SMITH and MARTIN M. SHAPIRO \\ Emory University, Atlanta, Georgia 30522
}

\begin{abstract}
The subjects, 108 4-year-old children from two socioeconomic levels, were trained on a three-phase simultaneous discrimination task. In Phase 1, stimulus element A was reinforced; stimulus element B was held constant. In Phase 2, element B was reinforced on all trials; element A was available and reinforced on $100 \%, 75 \%$, or $50 \%$ of the trials and held constant on the remaining trials. In Phase 3 , the two elements were tested separately, conjunctively, and disjunctively. Lower socioeconomic subjects (a) performed well on element A and blocked element B if element A was available on $100 \%$ of the Phase 2 trials, (b) performed poorly on both elements A and B if element A was $75 \%$ available, and (c) performed poorly on element $A$ and well on element $B$ if element $A$ was $50 \%$ available. Higher socioeconomic subjects performed well on both elements A and B under all conditions; however, predominant control passed from element $A$ to element $B$ after the availability of element $A$ had been reduced.
\end{abstract}

The term "blocking" was used by Kamin (1968) within the following paradigm: In Phase 1 , rats were trained to respond to stimulus element A; in Phase 2, rats were trained to respend to a simultaneous compound stimulus AB; and in Phase 3, stimulus elements A and B were tested separately. It was found that element $B$ had not acquired control over responding. Evidence of the phenomenon has also been found within operant conditioning. In general, blocking occurs if the experimental introduction of element $\mathrm{B}$ is not accompanied by a change in reinforcement, and blocking is circumvented if the introduction of element $\mathrm{B}$ is accompanied by $\mathrm{a}$ change in reinforcement.

Trabasso and Bower (1968) studied blocking in college students using a card-sorting task that was solvable along one stimulus dimension in Phase 1 and also solvable along an additional (redundant) dimension in Phase 2. They found that subjects learned nothing about the redundant cue if the initial solution still "worked" successfully. Their results were comparable to the results from animal experiments.

Anderson (1972) attempted to compare blocking and incidental learning in second-grade children. Unlike the other experiments, Anderson's procedure employed feedback, or reinforcement, during the Phase 3 test trials. Lyczak and Tighe (1975) obtained some blocking but concluded "that it is a low probability event ... and requires special testing conditions."

Requests for reprints should be addressed to J. L. Bresnahan, Department of Psychology, Herbert H. Lehman College, City University of New York, Bronx, New York 10468.
More extensive research efforts have been directed toward investigating the tendency of subjects to choose either reversal or extradimensional shifts when confronted with an optional shift procedure (e.g., Kendler \& Kendler, 1970; Tighe \& Tighe, 1966). In these optional shift studies, the correct stimulus choice of Phase 1 is incorrect in Phase 2, and reinforcement is used in Phase 3 ; in the blocking studies, a redundant stimulus is added in Phase 2 without shifting reinforcement from the previously reinforced stimulus, and no reinforcement is used in Phase 3. Although blocking is presumably related to other phenomena such as incidental learning and optional shifting, little or no direct evidence exists concerning blocking in children.

This experiment was designed to mimic the experimental procedure previously used to demonstrate blocking and to investigate procedures for attenuating the occurrence of blocking. For both purposes, children from two socioeconomic levels were used as subjects. Bresnahan and Shapiro (1972) had shown that children from a lower socioeconomic level preseverated on a stimulus dimension high on their hierarchy even when that stimulus dimension was not available on all trials. That is, the children did not attend to a second dimension which was available on all trials and which would have provided a solution on all trials, a result which could be interpreted as a form of blocking. Therefore, the design of this study included two socioeconomic levels and three experimental conditions. In one condition a redundant stimulus dimension was introduced in Phase 2 without reducing the availability of the orig- 
inally trained dimension; in two conditions the introduction of the redundant stimulus dimension was accompanied by reductions in the availability of the originally trained dimension.

\section{METHOD}

\section{Subjects}

The subjects were 108 preschool children selected from Atlanta area day care centers. A higher socioeconomic group composed of 33 girls and 21 boys (mean age $=4.33$ years) was selected from day care centers which required a $\$ 27$ per week tuition. A lower socioeconomic group composed of 30 girls and 24 boys (mean age $=4.44$ years) was selected from publicly supported day care centers. The children within each socioeconomic level were randomly assigned to the experimental groups.

\section{Apparatus}

The apparatus was the same as that used in previous studies (e.g., Bresnahan \& Shapiro, 1972) and has been described in detail elsewhere (Bresnahan, 1966). The subject was seated directly in front of a table on which rested a vertical panel, 61 by $70 \mathrm{~cm}$, painted with diagonal red and white stripes. Two cartoon faces, a smiling one and a sad one with tears, were inserted in the panel. Resting on the table within an opening in the panel was a royal-blue plywood tray. The tray could be completely covered or opened. A dark green chute also protruded from an adjoining panel. The experimenter served as the transducer, translating the subject's response into an electrical impulse by pressing a pushbutton switch. An easily discriminable bell and buzzer were mounted on the rear of the apparatus.

The stimuli were common clothing buttons. Two round yellow buttons were used: a smaller 2.6 -cm-diam button (SY) and a larger $3.5-\mathrm{cm}$-diam button (LY). Similarly, there were two dark blue buttons of the same physical dimensions (SB and LB, respectively). It was, therefore, possible to discriminate between the buttons on the basis of either size or color.

\section{Procedure}

The experiment consisted of three phases. The first two phases involved experimental manipulations; the third phase was a test phase, and therefore constant for all three experimental conditions. In Phase 1 , subjects in all three conditions were trained on one stimulus dimension while the second stimulus dimension was held constant. The experimental manipulation was accomplished by training one-half of the subjects on size and one-half of the subjects on color. All subjects were given the following instructions before Phase 1 began. "We're going to play a game. In this blue tray [experimenter pointing] there will be two things like this [experimenter dropping two practice stimuli, a gold belt buckle and a silver belt buckle, into the tray]. One of them is right; the other is wrong. I want you to pick the one you think is right and put it in the green chute here [pointing]. If you put the right one in the chute, this happy face will light up and a bell will ring [demonstration of light and bell]. If you put in the wrong one, this sad face will light up [pointing] and a buzzer will buzz [demonstration of light and buzzer]. Now, you put one in for practice. [After the subject places either of the two stimuli in the chute, the experimenter presses the "right" pushbutton.] That one was right, so the happy face came on. Now try these twn [experimenter dropping a different pair of stimuli, a tan wooden spherical button and a brown wooden football-shaped button, into the tray]. After the subject places either of the two stimuli in the chute, the experimenter presses the "wrong" pushbutton.] That one was wrong, so the sad face came on. When you pick the wrong one, go ahead and put the other one in the chute. [After the subject places the other one in the chute, the "right" pushbutton is pressed.] You understand how to play now, don't you? You want the happy face to come on as many times as you can; you don't want the sad face to come on. You want to get as many right as you can. All right, we'll begin now."

Subjects trained on size were presented with two different pairs of stimuli, SY-LY and SB-LB, in an unsystematic order. Small (S) was always the correct choice from the identically colored pair of stimuli. Subjects trained on color were also presented two pairs of stimuli, LY-LB and SY-SB, in an unsystematic order. Yellow (Y) was always the correct choice from the identically sized pair of buttons. Phase 1 was run for 16 trials, 8 trials with each pair.

Phase 2 was begun on Trial 17 without interruption. In Phase 2 , the dimension that was held constant within each pair of stimuli in Phase 1 was'made available as a discriminable dimension on every trial. The three experimental conditions varied in the proportion of trials on which the originally trained stimulus dimension was also available. Condition 1 constituted a typical blocking paradigm; the original stimulus dimension was also available on $100 \%$ of the trials. All 16 trials consisted of an SY-LB pair of buttons in which SY was always correct. Subjects originally trained to select the $Y$ color in Phase 1 could select SY in Phase 2 on the basis of $\mathrm{Y}, \mathrm{S}$, or both $\mathrm{S}$ and $\mathrm{Y}$; subjects originally trained to select the $S$ size in Phase 1 could also select SY in Phase 2 on the basis of Y, S. or both S and Y. Condition 2 was designed to attenuate blocking; the original stimulus dimension was discriminably available on only $75 \%$ of the trials. Twelve of the 16 trials consisted of an SY-LB pair of buttons in which SY was always correct. Subjects originally trained to select the $Y$ color received two trials of SY-LY and two trials of SB-LB, with S correct, unsystematically interspersed within the 16 trials. These subjects could obtain 12 correct choices in Phase 2 on the basis of Y, plus any lucky choices on the remaining four trials; alternatively, they could obtain 16 correct choices in Phase 2 on the basis of $S$ alone, or on the basis of both $S$ and $Y$. Subjects originally trained on the $S$ size received 12 SY-LB trials, 2 SY-SB trials, and 2 LY-LB trials, with Y correct, in unsystematic order; these subjects could obtain 12 correct on the basis of S, plus chance correct, and 16 correct on the basis of $Y$ alone, or on the basis of both $Y$ and $S$. Condition 3 was designed to further attenuate blocking; the original stimulus dimension was discriminably available on only $50 \%$ of the trials. Eight of the 16 trials consisted of an SY-LB pair in which SY was always correct. Subjects originally trained to select $Y$ received 4 SY-LY and 4 SB-LB trials, with S correct, interspersed within the 16 trials. Subjects originally trained to select $S$ received 4 SY-SB and 4 LY-LB trials, with Y correct, interspersed within the 16 trials. The subjects in Condition 3 could obtain eight correct choices on the basis of the original dimension, plus any lucky choices on the remaining eight trials; alternatively, they could obtain 16 correct choices either by attending only to the new stimulus dimension or by attending to both stimulus dimensions.

Following the completion of Phase 2, the subject was given the following additional instructions. "The happy face and the sad face will no longer light up, and the bell and the buzzer will no longer sound. Instead, I'm going to keep score. I want you to keep putting the button that would give you the happy face in the green chute. If you do well, you'll win a prize."

A total of 48 trials were run during Phase 3, the test phase. Four types of test trials were used: (a) color and size positively related (SY-LB), (b) color and size negatively related (LY-SB), (c) both buttons of the same color, but different size (SY-LY or SB-LB), and (d) both buttons of the same size, but different color (LY-LB or SY-SB). Twelve trials of each type were run; every four trials contained one trial of each type. When there were two kinds of trials that satisfied a type [i.e., $c$ and $d$ above ], six trials of each kind were run. The order of trials was unsystematic and was the same for all subjects. 


\section{RESULTS}

The dependent variables were the choice of stimuli on the four types of test trials.

\section{Test Trials with Consistent and \\ Redundant Stimulus Values}

An analysis of variance of the number of SY choices on the 12 test trials with SY-LB yielded no statistically significant effects or interactions. These test trials constituted an overall measure of learning in Phases 1 and 2; SY was the correct choice for subjects choosing on the basis of the Y color, the S size, or the SY compound. The null hypothesis that all groups learned equally well to choose SY was therefore not rejected.

\section{Test Trials with Conflicting}

\section{Stimulus Values}

A 2 by 2 by 3 (Phase 1 Training Dimension by Socioeconomic Level by Phase 2 Percentage of Availability of the Original Dimension) analysis of variance of the number of choices of the originally trained stimulus on the 12 LY-SB test trials was calculated. The results shown in Figure 1 demonstrate that the number of choices of the originally trained stimulus decreased as the Phase 2 availability of that stimulus dimension decreased from $100 \%$ to $75 \%$ to $50 \%, F(2,96)=13.00$, $\mathrm{p}<.01$. A significant interaction between the Phase 1 dimension, color or size, and the Phase 2 availability of that dimension, $F(2,96)=3.14, p<.05$, resulted primarily from the finding in the $50 \%$ condition that subjects originally trained on size chose the LY and SB stimulus buttons equally often and that subjects originally trained on color chose the appropriately colored stimulus (LY) significantly less often than expected by chance. (Chance was defined by the $95 \%$ confidence interval.) That is, subjects in the $50 \%$ condition, who had been trained on color in Phase 1, significantly chose the appropriately sized stimulus on the test trials.

\section{Separate Test Trials of the Stimulus Dimension \\ Trained in Phase 1 and the Stimulus \\ Dimension Trained in Phase 2}

The dependent variable was the number of appropriate choices on the color test trials and the size test trials. For each group of subjects, one of these dimensions had been trained in Phase 1 and one had been trained in Phase 2. A mixed-design analysis of variance was computed, with choices on test trials of the firstand second-trained dimensions as a within-subject variable and socioeconomic level, Phase 1 training dimension, and Phase 2 availability of that dimension as between-subject variables. The results are shown in Figures 2 and 3 . It can be seen in Figure 2 that subjects from the higher socioeconomic level performed significantly better on both dimensions than children from the lower socioeconomic level, $F(1,96)=7.12, p<.01$. A significant interaction between Phase 2 availability and performance on the first- and second-trained dimensions,

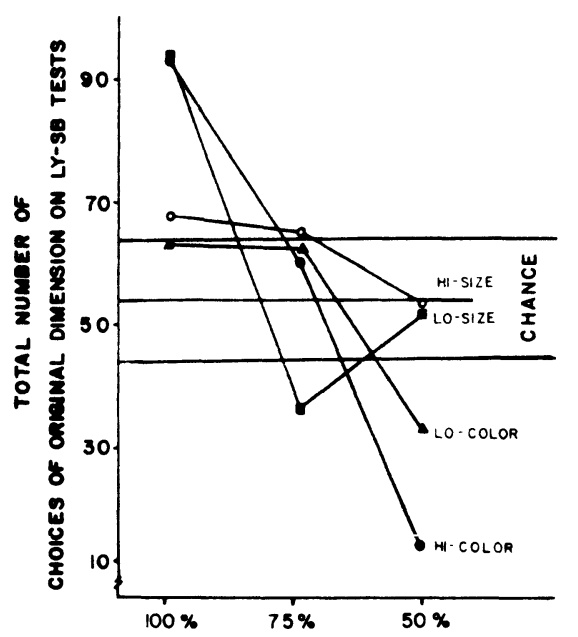

PHASE \| AVAILABILITY OF PRETRAINED DIMENSION

Figure 1. Number of choices of stimulus originally trained in Phase 1 on LY-SB test trials in Phase 3. Each curve represents the results for a higher or lower socioeconomic group trained on color or size in Phase 1.

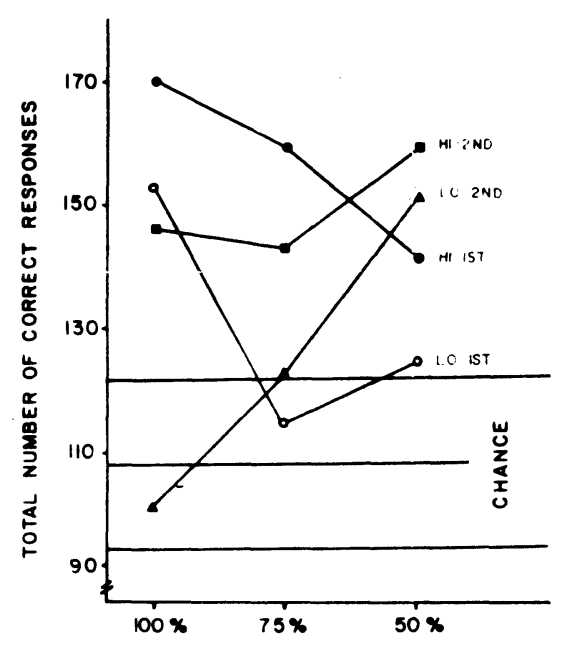

PHASE II AVAILABILITY OF PRE TRAINED DIMENSION

Figure 2. Number of correct choices on the first-trained and the second-trained dimensions tested separately in Phase 3, as a function of socioeconomic level.

$F(2,96)=6.61, p<.01$, is evident in the crossing curves for first- and second-trained dimensions for both higher and lower socioeconomic groups in Figure 2. Higher socioeconomic level subjects, who always performed better than chance, were more accurate on the first dimension than on the second unless the Phase 2 availability of the first dimension was reduced to $50 \%$. Lower socioeconomic level subjects, who did not always perform better than chance, were more accurate on the first dimension than on the second if the first dimension was available on $100 \%$ of the trials in Phase 2; they were more accurate on the second dimension than on the first if the first dimension was available on only $50 \%$ of the trials in Phase 2.

A triple interaction among Phase 1 dimension (color 


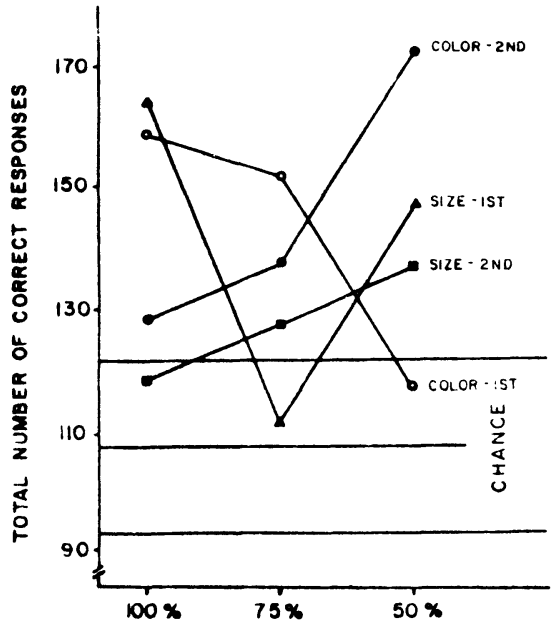

PHASE \| AVALLABIL.ITY OF PRE TRAINED DIMENSION

Figure 3. Number of correct choices on the first-trained and the second-trained dimensions tested separately in Phase 3, as a function of stimulus dimensions trained in Phase 1. The curves labeled Color-1st and Color-2nd represent the results from the subjects trained on color in Phase 1 and subsequently tested on color and size, respectively. The curves labeled Size-1st and Size-2nd represent the results from the subjects trained on size in Phase 1 and subsequently tested on size and color, respectively.

or size), Phase 2 availability, and performance on firsttrained and second-trained dimensions, $F(2,96)=3.42$, $\mathrm{p}<.05$, is shown in Figure 3. Subjects first trained on color performed better on the first dimension than on the second unless color was only $50 \%$ available in Phase 2 (curves labeled Color-1st and Color-2nd). Subjects trained on size first did not produce as clear a result. These subjects went from a chance level of performance on their second dimension in the $100 \%$ condition to higher levels of performance on their second dimension as the Phase 2 availability of the size decreased to $75 \%$ and $50 \%$. Their performance on the first-trained size dimension was not a monotonic function of the Phase 2 availability of size.

\section{DISCUSSION}

The performance of the subjects from the higher socioeconomic level will be discussed first. Subjects initially trained on size showed relatively little preference for size over color on forced-choice trials, whereas subjects initially trained on color showed a large preference for color after it was $100 \%$ available in Phase 2 and a large rejection of color after it was $50 \%$ available in Phase 2 (Figure 1). Under all three experimental conditions, higher socioeconomic subjects performed well on both the first- and second-trained dimensions when they were tested separately (Figure 2). In general, higher socioeconomic subjects did not display blocking in the $100 \%$ condition although, as expected, the relative behavioral control exerted by the firsttrained dimension was greatest in the $100 \%$ condition and smallest in the $50 \%$ condition. Subjects initially trained on color displayed a more robust effect than subjects initially trained on size.

The performance of subjects from the lower socioeconomic level was quite different. Subjects initially trained on size showed a large preference for the first-trained dimension after $100 \%$ availability in Phase 2 (Figure 1). The results from the test trials on which the first- and second-trained dimensions were tested separately (Figure 2) show that these subjects performed well on the first-trained dimension after $100 \%$ availability and responded at a chance level on the second dimension under $100 \%$ condition. The lower socioeconomic subjects displayed blocking of the redundant dimension. Furthermore, reduction in availability of the first-trained dimension, from $100 \%$ to $75 \%$ and $50 \%$, produced very poor performance on the first-trained dimension; $50 \%$ availability of the first-trained dimension produced good behavioral control by the second-trained dimension. In general, subjects from the lower socioeconomic level displayed blocking under the appropriate condition, displayed little stimulus control in the $75 \%$ condition, and shifted stimulus control almost completely to the second stimulus in the $50 \%$ condition. Strong stimulus control was never exerted by both stimulus elements under any condition. The subjects appear to have consistently attended to only one stimulus element, at most. This result is in sharp contrast to the behavior of the higher socioeconomic subjects, who consistently learned both stimulus elements.

Four-year-old subjects from a higher socioeconomic level did not display blocking found in either animals or adult human beings; lower socioeconomic subjects did display the blocking. As in animal studies, the blocking may be prevented by changing the reinforcement contingency coincident with the introduction of the new stimulus dimension.

However, interpretation of the obtained socioeconomic effect is difficult. A related result in the literature indicates that performance on incidental learning tasks is a nonmonotonic function of age (Siegel, 1968; Siegel \& Stevenson, 1966). Similarly, given the results of this experiment, one might conjecture that blocking is a nonmonotonic function of age.

\section{REFERENCES}

ANDERSON, D. R. The effects of prior training on the incidental discriminative learning of children. Journal of Experimental Child Psychology, 1972, 14, 416-426.

Bresnahan, J. L. The effect of task and incentive on concept acquisition with children from two socioeconomic levels (Doctoral dissertation, Emory University, 1966). Dissertation Abstracts International, 1967, 27, 2886B. (University Microfilms No. 67-762).

Bresnahan, J. L., \& Shapiro, M. M. Learning strategies in children from different socioeconomic levels. In H. W. Reese (Ed.), Advances in child development and behavior (Vol. 7). New York: Academic Press, 1972.

Crane, N. L., \& Ross, L. E. A developmental study of attention to cue redundancy introduced following discrimination learning. Journal of Experimental Child Psychology, 1967, 5, 1-15.

Kamin, L. J. "Attention-like" processes in classical conditioning. In M. R. Jones (Ed.), Miami symposium on the prediction of behavior: Aversive stimulation. Miami: University of Miami Press, 1968.

Kendler, H. H., \& KendLeR, T. S. Vertical and horizontal processes in problem solving. Psychological Review, 1962, 69, $1-16$.

KendleR, T. S., \& KendleR, H. H. An ontogeny of optional shift behavior. Child Development, 1970, 41, 1-27.

LyCZAK, R., \& Tighe, T. Stimulus control in children under a blocking paradigm. Child Development, 1975, 46, 115-122.

SIEgel, A. W. Variables affecting incidental learning in children. Child Development, 1968, 39, 957-968.

Siegel, A. W., \& Stevenson, H. W. Incidental learning: A developmental study. Child Development, 1966, 37, 811-818.

Tighe, T. J., \& Tighe, L. S. Overtraining and optional shift behavior in rats and children. Journal of Comparative and Physiological Psychology, 1966, 62, 49-54.

Trabasso, T., \& Bower, G. H. What is learned. In T. Trabasso \& G. H. Bower (Eds.), Attention in learning: Theory and research. New York: Wiley, 1968.

(Received for publication March 26, 1976.) 